Contributors NAA conceived the study idea and is PI of the SLOPE (Studying Lifecourse Obesity PrEdictors) study. NZ cleaned and managed the raw data. EJT analysed the data. EJT drafted the abstract. All authors contributed to the study design, interpreted the results, revised the abstract for content and approved the final version before submission.

Acknowledgements David Cable (Electronic Patient Records Implementation and Service Manager) and Florina Borca (Senior Information Analyst R\&D) at University Hospital Southampton NHS Foundation Trust for support in accessing the data used in this study.

\section{OP65 PREDICTION MODELS FOR POPULATION TOBACCO USE: A SYSTEMATIC METHODOLOGICAL REVIEW TO IDENTIFY BEST MODELLING STRATEGIES}

V Huang*, A Head, L Hyseni, M O'Flaherty, I Buchan, S Capewell, C Kypridemos. Public Health and Policy, University of Liverpool, Liverpool, UK

10.1136/jech-2020-SSMabstracts.64

Background Smoking remains a top public health priority, killing over $6 \mathrm{~m}$ people annually. Planning future tobacco control policies can greatly benefit from population prediction models for tobacco use (mathematical models that simulate tobacco exposure and its health impact in the population). Those models were mainly developed to project trends and simulate policy as identified in a systematic review published in 2013. Common outcomes were changes in tobacco use behaviour, tobacco-related morbidity/mortality, and economic impact. We updated, expanded and enhanced the 2013 review. We aimed to identify best modelling practices, highlight common pitfalls, and develop a quality-assessment checklist.

Methods We systematically searched PubMed, Embase, CINAHL Plus, EconLit, and PsycINFO for publications between July 2013 and August 2019 using the search strategy of the 2013 review. We included studies referring to tobacco product or tobacco use and projected a tobacco-related outcome. We only included studies in English. Two reviewers independently assessed the eligibility of the identified studies through title and abstract screening followed by full-text review; all discrepancies were resolved in consensus with a third reviewer. We designed and piloted a data-extraction form based on existing guidelines to collect information such as model structure, data sources and transparency. We analysed the evidence using narrative synthesis. We developed a qualityassessment checklist for population prediction tobacco models, including the risk of bias and standard quality criteria.

Results In total, 5046 records were identified of which 830 were duplicates; 80 papers were included in this review. A diverse range of modelling/simulation methodologies, including microsimulations, decision-trees, and agent-based models have been used in population tobacco use prediction modelling. However, methodological transparency was notably lacking. Furthermore, the tobacco modelling community apparently works mostly in 'silos', hindering the diffusion of good modelling practice, and promoting wasteful repetition of effort. For example, while some models appropriately simulate smoking intensity and duration to model cumulative hazard, others only simulate smoking status (i.e. never/ever/current).

Conversely, the modelling teams participating in the Cancer Intervention and Surveillance Modelling Network (CISNET) collaborated well, sharing data, methodological advancements and 'building blocks' for their models.

Worryingly, some tobacco models received industry funding, making bias likely.

Conclusion Population prediction modelling for tobacco use is an active area of research. However, our systematic methodological review identified variable quality and an overall lack of transparency. More active collaboration using transparent methods and open-source code could avoid wasteful duplication of effort, speed scientific progress and benefit both the tobacco control community and wider society.

\section{OP66 OPINIONS AND EXPERIENCES OF A NATIONAL SMOKEFREE PRISON POLICY: EVIDENCE FROM THE TOBACCO IN PRISONS STUDY}

${ }^{1} \mathrm{~A}$ Brown*, ${ }^{2} \mathrm{H}$ Sweeting, ${ }^{1} \mathrm{~K}$ Hunt. ${ }^{1}$ Institute for Social Marketing and Health, University of Stirling, Stirling, UK; ${ }^{2} \mathrm{MRC} / \mathrm{CSO}$ Social and Public Health Sciences Unit, University of Glasgow, Glasgow, UK

\subsection{6/jech-2020-SSMabstracts.65}

Background A national smokefree prison policy was implemented in Scotland from November 2018. The removal of tobacco from a prison system poses some distinct potential challenges, not least because prisons are 'homes' and rates of smoking in the prison population are very high. The Tobacco In Prisons study (TIPs) is internationally unique in comprehensively studying the introduction of restrictions on smoking in Scottish prisons, using data collected before, during and after the change to prison smoking policy. This paper presents opinions and experiences of prison smoking restrictions among people in custody (prisoners) and staff in Scotland, and identifies implementation success factors and lessons that are highly relevant for other jurisdictions and areas of public health.

Methods Surveys of staff (online) and people in custody (paper-based) were conducted in November-December 2016 (return rates: 26.6\% (staff); 33.8\% (people in custody)); May-July 2018 (31.4\%; 25.9\%) May-July 2019 (16.1\%; $18.1 \%)$. The surveys contained identical or similar questions on topics related to smoking, smoking cessation/abstinence and smoking restrictions in the prison context. Topics were also explored qualitatively with staff and people in custody at similar time points to the surveys via 34 focus groups and 99 interviews in total. Changes in survey responses over time were analysed using descriptive statistics and logistic regression analyses, adjusting for potentially confounding socio-demographic variables. Qualitative data were thematically analysed to identify the diversity of views and experiences.

Results The new smokefree policy is widely accepted as the new 'norm' in Scottish prisons, although support was consistently higher among staff than people in custody before, during and after implementation. Both the surveys and qualitative work suggest that perceptions of some of the potential difficulties ('hard to enforce') and negative consequences ('cause a lot of trouble') of a smokefree prison policy reduced post implementation. Participants identified several implementation success factors relating to: planning and communication, smoking abstinence/cessation products/services, and partnership working.

Conclusion Our study confirms that smokefree prison polices can be successfully implemented, despite widespread 